\title{
The Relationship Between the Metabolic Syndrome and the Place of Residence in the Local Community on the Example of the Janów Lubelski District in Eastern Poland: A Population-Based Study
}

\author{
Grzegorz Józef Nowicki $\mathbb{D}^{\prime}$ \\ Barbara Ślusarska (D) \\ Katarzyna Naylor (iD ${ }^{2}$ \\ Andrzej Prystupa ${ }^{3}$ \\ Ewa Rudnicka-Drożak ${ }^{4}$ \\ Ulyana Halyuk (iD) ${ }^{5}$ \\ Petro Pokotylo (iD ${ }^{5}$ \\ 'Department of Family Medicine and \\ Community Nursing, Medical University \\ of Lublin, Lublin, Poland; ${ }^{2}$ Department of \\ Didactics and Medical Simulation, Medical \\ University of Lublin, Lublin, Poland; \\ ${ }^{3}$ Department of Internal Medicine, \\ Medical University of Lublin, Lublin, \\ Poland; ${ }^{4}$ Department of Family Medicine, \\ Medical University of Lublin, Lublin, \\ Poland; ${ }^{5}$ Department of Normal \\ Anatomy, Lviv National Medical \\ University, Lviv, Ukraine
}

Correspondence: Grzegorz Józef Nowicki Department of Family Medicine and Community Nursing, Medical University of Lublin, Staszica 6 Street, PL-20-08I, Lublin, Poland

Tel +48 8I448 68I0

$\mathrm{Fax}+48814486811$

Email gnowicki84@gmail.com
Purpose: The aim of this study was to estimate the incidence concerning metabolic syndrome (MetS) in a local community with a low socioeconomic status and a medium and high cardiovascular risk on the example of residents of Janów Lubelski district, eastern Poland. The second goal of the research was to analyze the relationship between residence and the occurrence of MetS.

Methods: We conducted a cross-sectional study of 4040 people living in eastern Poland. A group of 3046 people with medium and high cardiovascular risk was selected among the respondents and included in further analyzes. The research adopted the definition criteria proposed by the National Cholesterol Education Program - Third Adult Treatment Panel (NCEP ATP III) to implement diagnostic evaluation of MetS.

Results: It was observed that metabolic syndrome was significantly more frequent among the inhabitants of rural areas $(40.56 \% ; n=810)$ compared to those living in the city $(35.27 \%$; $\mathrm{n}=370) \mathrm{p}=0.005$. Among the inhabitants of rural areas, the percentage of people with elevated glucose levels was significantly higher, fasting blood glucose (FGB) $p<0.001$, elevated blood pressure (HBP) $p<0.001$, elevated serum triglycerides (TGs) $p=0.01$, and abnormal waist circumference (WC) $\mathrm{p}=0.003$ compared to urban inhabitants. After adjusting for potential confounding variables (age, education, smoking, marital status, and level of physical activity), in both women and men, the odds of developing metabolic syndrome were approximately $30 \%$ higher in rural areas compared to urban residents (women: odds ratio $(\mathrm{OR})=1.25,95 \%$ confidence intervals $(\mathrm{CI})=1.01-1.56$; men: $\mathrm{OR}=1.30,95 \% \mathrm{CI}=1.01-1.67)$.

Conclusion and Recommendations: A higher incidence of metabolic syndrome was observed among respondents living in rural areas than those living in cities. Similarly, across the gender strata, metabolic syndrome is more commonly diagnosed among men and women living in rural areas. Healthcare workers, especially in rural areas, should engage in education, prevention, and the promotion of a healthy lifestyle.

Keywords: metabolic syndrome, rural, urban, prevalence, population-based study

\section{Introduction}

Metabolic syndrome (MetS) is a related complex of metabolic disorders, including abdominal obesity, elevated blood pressure (HBP), elevated fasting blood glucose (FBG) and dyslipidemia (elevated triglycerides (TGs) and/or reduced levels of high-density lipoprotein (HDL-C) cholesterol). ${ }^{1}$ It is a premorbid condition that develops when the following factors develop: insulin resistance, poor diet, physical 
inactivity, obesity and genetic burden, as they are considered to be essential cofactors of MetS. ${ }^{2}$ MetS is a major global public health challenge, especially in developing and developed countries, as it directly adds to the burden on health systems by contributing to type 2 diabetes (T2D), cardiovascular disease (CVD), ${ }^{3}$ and cancer. ${ }^{4}$ The prevalence of MetS in Europe is approximately $10.47 \%$ of the population and $16.04 \%$ in Africa, $21.27 \%$ in Asia, and $30 \%$ in the United States respectively. ${ }^{5}$

The prevalence of MetS in the adult Polish population remains unclear. In population studies conducted in Poland, by the Multicentre National Population Health Examination Survey (WOBASZ), MetS was recognised in $23.9 \%$ of women and $26 \%$ of men (according to the definition of the American Heart Association (AHA)/ National Heart, Lung, and Blood Institute (NHLBI) 2005 ) and in $26.8 \%$ of women and $30.7 \%$ of men (as defined by the International Diabetes Federation (IDF) 2005). ${ }^{6}$ However, in the IDF definition, central obesity was recognized as an essential component of MetS. Simultaneously, in the US, the suggested waist cut off value was higher than currently accepted for Europeans. In the Prospective Urban and Rural Epidemiological Study Poland (PURE), MetS was diagnosed in $44.1 \%$ of the respondents, of which $41.8 \%$ were female and $48.2 \%$ male. Moreover, it was found that in the group of people with MetS, there were more elderly and rural residents and fewer people with a higher education. ${ }^{7}$ The Polish Norwegian Study (PONS) revealed that in the Polish population, the prevalence of MetS was $39.5 \%$ in the general population. Additionally, the frequency of MetS among women was $34.3 \%$, and among men - $49.9 \%{ }^{8}$ However, due to the changing diagnostic criteria of MetS over time, it is challenging to compare the epidemiological data on the prevalence of the disorder. Nevertheless, the percentage of people who develop symptoms of MetS is increasingly high. ${ }^{9}$ There is a lack of research, especially in Central and Eastern European countries, on the spread of MetS.

Environmental factors, including urbanization and the westernization of lifestyles, can significantly influence the prevalence of MetS in different population groups. The incidence of MetS is influenced by socioeconomic factors, such as education and income, as well as lifestyle factors, such as diet, smoking, alcohol consumption and physical activity. ${ }^{10}$ It is believed that economic progress leads to a higher prevalence of atherogenic diets in the general population, decreased physical activity, increased smoking and alcohol consumption and an increase in the percentage of overweight and obese people, which directly translates into the presence of MetS. ${ }^{11}$ Urbanization and economic progress and also the westernization of life, are affecting more and more people in rural areas, which has an impact on the prevalence of MetS. However, epidemiological analyses do not indicate a higher proportion of people with MetS in rural or urban areas. India, ${ }^{12}$ China, ${ }^{13}$ Malaysia $^{14}$ and sub-Saharan Africans ${ }^{15}$ have found MetS to be more common in cities than in rural areas. Thus, it can be argued that as the rural community moves away from urbanization, lifestyle changes and associated diseases are more pronounced in urban areas. In contrast, studies in the United States, ${ }^{16}$ Korea $^{17}$ and South Africa ${ }^{18}$ have found a higher prevalence of MetS among rural residents. Differences in MetS occurrence between rural and urban residents can be attributed to differences in demographic and socio-cultural characteristics. ${ }^{19}$ Interesting observations were made by Yang et al, ${ }^{20}$ assessing the relationship between living in areas with more and less vegetation and the presence of MetS. They observed that living in greener areas was associated with a lower percentage of people with MetS, especially among those under the age of 65 and with a higher household income.

The study aims at estimating the prevalence of the metabolic syndrome in a local community with a low socioeconomic status and a medium and high cardiovascular risk, according to the Systematic Coronary Risk Evaluation (SCORE) scale based on the example of residents of the Janów Lubelski district, eastern Poland. The second goal of the research was to analyse the relationship between the place of residence and the presence of MetS.

\section{Materials and Methods Study Design and Participants}

The prevention and health promotion program in the field of cardiovascular diseases, entitled "Follow Your Heart", was conducted in the period from June 14, 2015, to March 20, 2016, among the inhabitants of the Janów Lubelski district in the south-western part of Lublin voivodeship, which lies in Eastern Poland (Figure 1). The implementation of the above project was financed by the Norwegian Financial Mechanism 2009-2014 under the PL Program 13 "Limiting social inequities in health". The procedure could be entered as research in local communities with high standardized mortality rates (SMRs), 
A

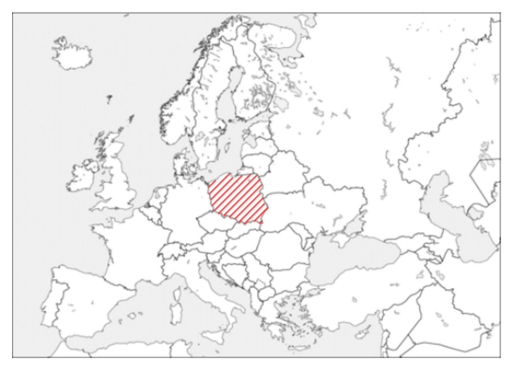

B

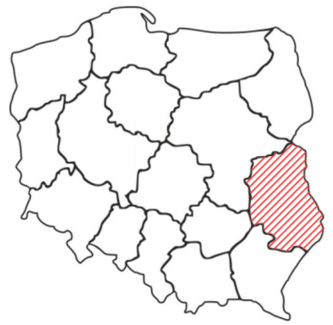

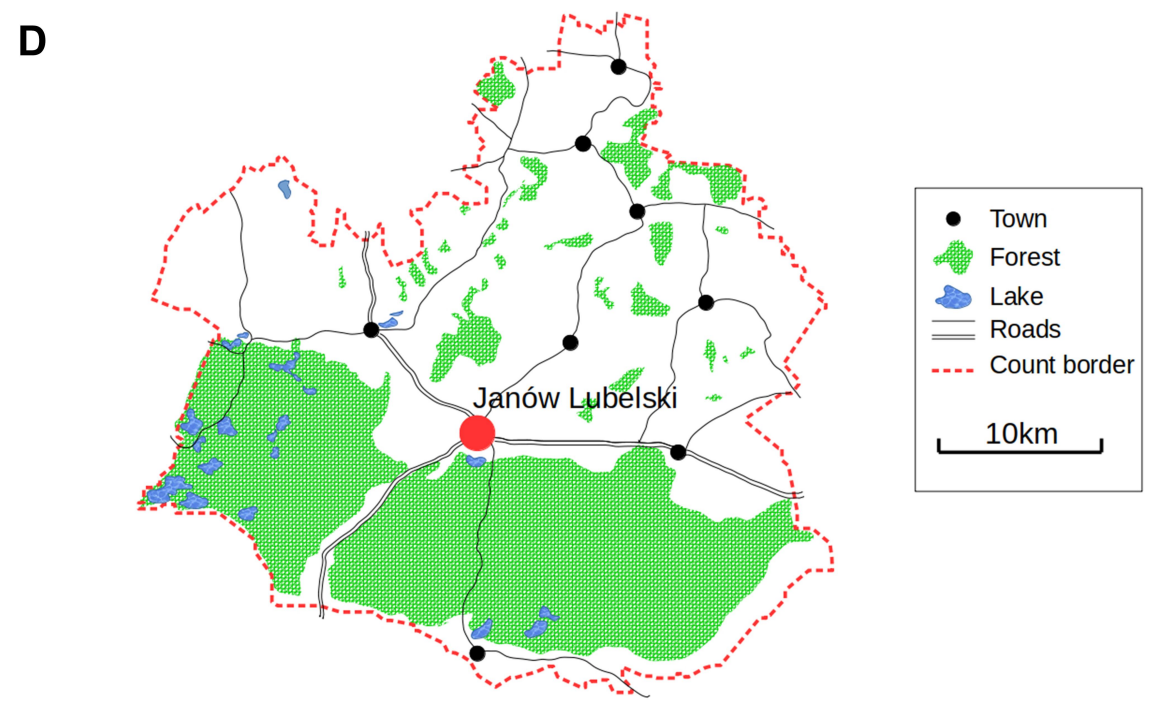

\section{C}
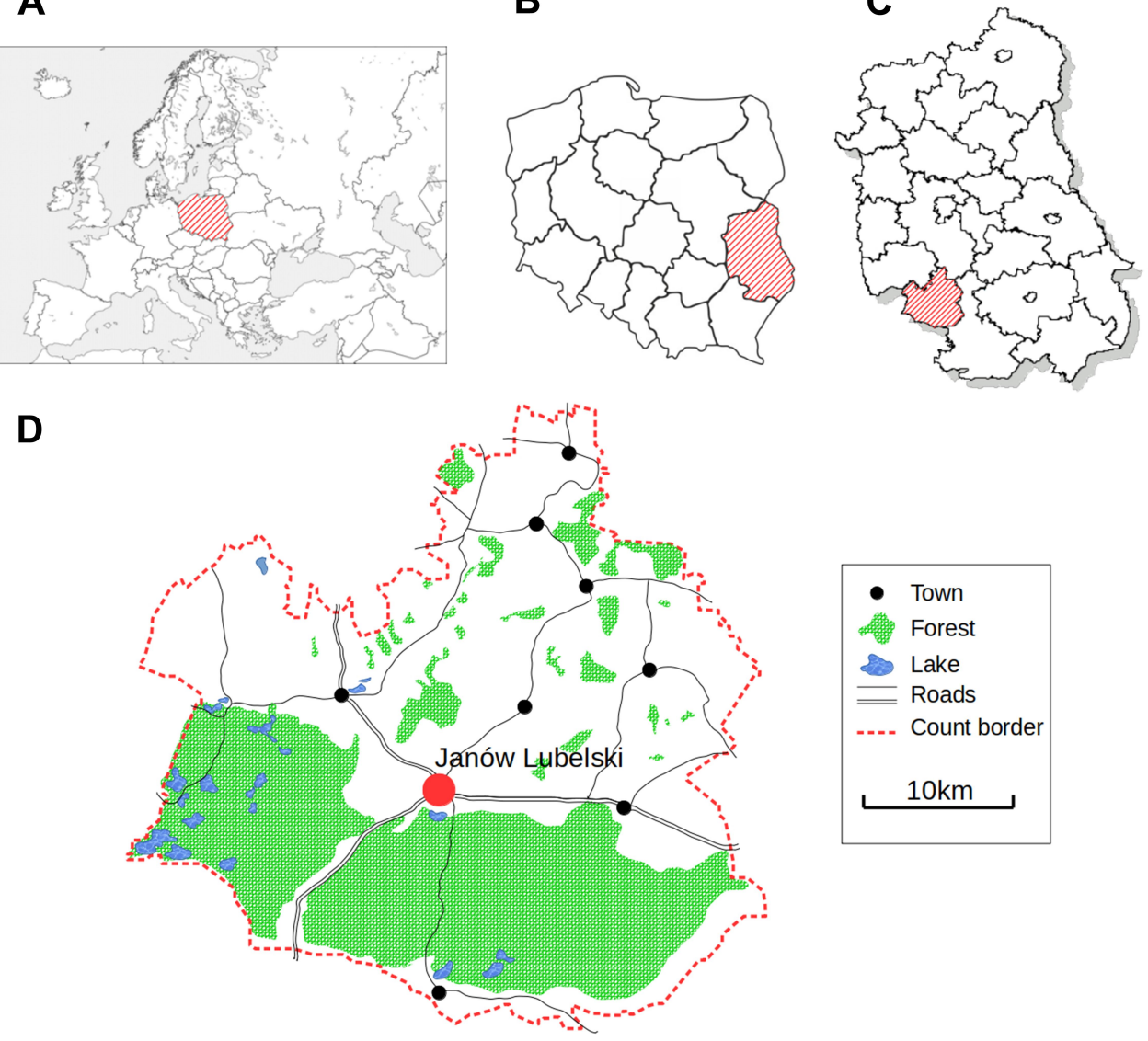

Figure I Approximate residential locations of participants, Janow Lubelski district, Poland.

Notes: (A) Europe; (B) Poland; (C) Lubelskie Voivodeship; (D), Janów Lubelski District

including those from cardiovascular causes. The list of districts eligible to participate in the competition was developed based on the highest SMR in 2009-2011 in the following categories: malignant neoplasms, CVD, respiratory system diseases, digestive system diseases, external causes and total mortality. The Janów Lubelski district was included on the list in the third position ( $\mathrm{SMR}=1.357$ ) among 38 enlisted, with the highest standardized mortality rates due to CVD in Poland. It was characterized by one of the lowest ranks according to the human development index (HDI) among districts in Poland. $^{21,22}$ In the period preceding the survey, 47,500 people were living in the area of Janów Lubelski. ${ }^{23}$ The group participating in the project consisted of respondents aged 35-64; an eligible population of 18,827 Janów Lubelski residents according to the population records and electoral lists. The recruitment of respondents for research and its promotion were conducted through the district and commune local governments and cooperating institutions (religious unions, workplaces, associations, and public utility organizations) and telephone invitations to persons from the lists eligible for the research. In order to ensure equal access to the study, 15 points were organized in Janow Lubelski district, where the respondents could report (14 mobile -touring points, located in various towns and one stationary point, located in the City Hospital, which also had a coordinating function). Four thousand forty people benefited from invitations and voluntary tests; the overall participation rate was $21.45 \%$ of the eligible population. The study included people aged 35 to 64 , residents of Janów Lubelski district who arrived to the research point and provided their informed consent for the study. The exclusion criteria included: those aged under 35 and over 64, people unable to give informed consent, bedridden residents and those living in nursing homes and prisons. The study includes people under the age of 65. It is essential to establish the prevalence of MetS components in younger age groups, as the presence of MetS components may indicate an increased risk of CVD later in life. Besides, an early identification of the 
components of MetS offers the opportunity to undertake targeted interventions to prevent the development of the syndrome and thus reduce the risk of CVD later in life. ${ }^{24}$ The study consisted of two stages. The first stage - screening - covered 4040 people. Based on the results obtained in stage I (assessment of cardiovascular risk using SCORE, which, based on information about age, smoking, systolic blood pressure and total cholesterol, estimates the 10 -year risk of death from $\mathrm{CVD}^{25}$ ), people with a medium and high risk of SCORE $\geq 5$ were qualified for the second stage. Three thousand one hundred seventy-nine people were qualified for the second stage. However, due to a history of a cardiovascular incident (myocardial infarction or stroke), 133 respondents were excluded from the analysis (Figure 2). Data on the occurrence of cardiovascular events were collected in a personal history.

\section{Data Collection}

The data collection team consisted of specially trained nurses. Socio-demographic data (age, gender, place of residence, education, smoking status assessment and physical activity assessment) were collected using standard questionnaires. A cigarette smoker was defined as a respondent who smoked at least one cigarette in the previous month. ${ }^{26}$ The assessment of physical activity was based on information received through an interview concerning whether the respondent performed regular physical activity at least five times a week for 30 minutes.

An inelastic tape measure was used to measure the waist circumference (WC) - between the lower edge of the costal arch and the iliac bone's upper crest. Measurements were made in a standing position with an accuracy of $0.5 \mathrm{~cm}^{27}$

The participants of the study underwent measurements of blood pressure (BP) twice, including systolic blood pressure (SBP) and diastolic blood pressure (DBP). Measurements were taken on the left arm using an electronic sphygmomanometer. The first BP measurement was taken after at least 5 minutes' rest and another 15 minutes after the first one. The mean value of the two measurements was analysed. If both measurements differed by more than $5 \mathrm{~mm} \mathrm{Hg}$, an additional measurement was taken after another 15 minutes and the average of the three measurements was used for data analysis. ${ }^{28}$

Blood samples were taken from the ulnar vein in the morning on an empty stomach after an overnight rest, put into a tube with a clotting activator and a separating agent (granules) and then delivered to the laboratory within 4 hours. Plasma was separated by centrifugation at $3000 \mathrm{rpm}$ for 10 minutes. Centrifuged serum was used to determine blood glucose, TGs, and HDL-C. FBG was measured by the enzymatic-hexokinase method. The concentration of TGs was obtained by the enzymatic colorimetric method,

Study Design

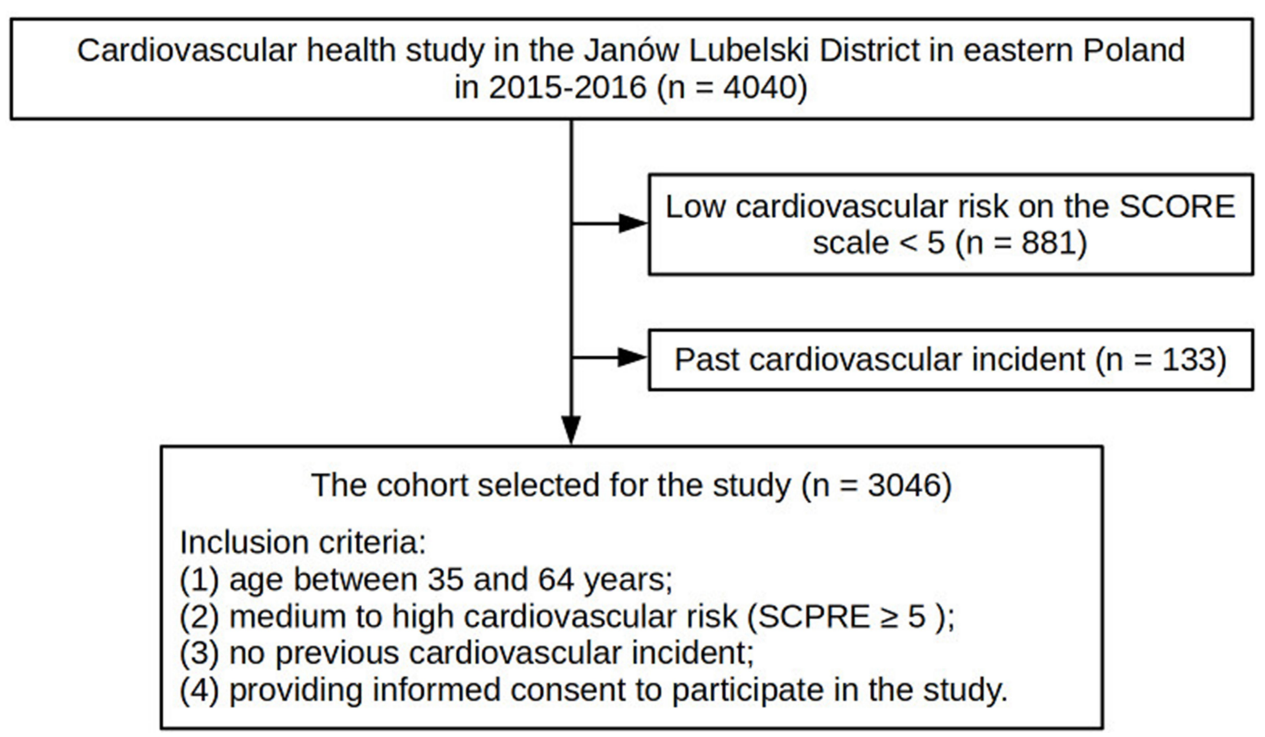

Figure 2 Study design. 
while HDL-C through the homogeneous colorimetric enzymatic method. Both parameters were determined on an Advia 1800 or Advia 1200 apparatus using Siemens reagents. The markings were made at the Central Laboratory of the hospital in Janów Lubelski.

\section{Definitions of MetS}

The research adopted the definition proposed by the National Cholesterol Education Program - Third Adult Treatment Panel (NCEP ATP III). ${ }^{29}$ According to the definition, the presence of at least three of the five risk factors is the basis for the diagnosis of metabolic syndrome, including: $\mathrm{FBG} \geq 100 \mathrm{mg} / \mathrm{dL}, \mathrm{HBP}$ (SBP $\geq 130 \mathrm{mmHg}$ and $\mathrm{DBP} \geq 85 \mathrm{mmHg}$ ), TGs $\geq 150 \mathrm{mg} / \mathrm{dL}$, decreased HDL-C concentration (women $<50 \mathrm{mg} / \mathrm{dL}$ and men $<$ $40 \mathrm{mg} / \mathrm{dL}$ ) and elevated $\mathrm{WC}$ (women $>88 \mathrm{~cm}$ and men $>102 \mathrm{~cm}$ ). When choosing the MetS definition, we were guided by the fact that the study group was characterized by medium and high cardiovascular risk. In the Polish population, there are no results of studies comparing the definitional differences in MetS. Therefore, we have referred to the results obtained by Nilsson et $\mathrm{al}^{30}$ among Caucasians. Their definition of MetS was superior in predicting cardiovascular events in the case of both genders compared to the definition of The International Diabetes Federation (IDF). ${ }^{31}$ Moreover, research suggest that the waist circumference cut-off in the IDF definition may be too low in the Caucasian population, ${ }^{32}$ thereby leading to overdiagnosis of MetS. ${ }^{33}$ Also, the results of The International Day for Evaluation of Abdominal Obesity study (IDEA) on hypertensive patients in the Polish population compared the definitional differences according to the ATP III and IDF criteria for abdominal obesity in the Polish population. The IDEA indicated that the IDF criteria lead to an increase in the incidence of abdominal obesity in patients with arterial hypertension from $67.2 \%$ to $86.9 \%$. $^{34}$

\section{Statistical Analysis}

The distribution of categorical variables was presented as numbers and percentages, as means with standard deviations for normally distributed continuous variables. The Kolmogorov-Smirnov test was used to verify the normality of the data distribution. Continuous variables were compared using a Student's $t$-test and categorical ones with a chi-square test. The multivariable logistic regression model was used to investigate the association between MetS' prevalence and place of residence. The analyses were conducted separately for men and women. The logistic regression model results were presented in an odds ratio (OR) and 95\% confidence intervals $(\mathrm{CI})$. All analyses were performed by IBM Corp. Released in 2019. IBM SPSS Statistics for Windows, Version 26.0. Armonk, NY: IBM Corp. Statistical significance was defined as $\mathrm{p}<0.05$.

\section{Results \\ Identification and Characteristics of the Participants}

Among the 4040 original participants at the baseline examination in the open cohort, 3046 eligible subjects were included in the study. Eight hundred eighty-one subjects with low SCORE cardiovascular risk $(<5)$ and 133 subjects with a history of a cardiovascular event (eg, myocardial infarction, stroke, etc.) were excluded from the study. Compared to the excluded participants, the respondents included in the research were older and had a lower education. The remaining features did not differentiate between the groups of included and excluded participants (Supplementary Table S1).

\section{General Characteristics of Participants}

Table 1 presents the study group's characteristics in strata of the place of residence and gender. The majority of the studied group were females $(57.9 \% ; n=1765)$ and people living in rural areas $(65.6 \% ; n=1998)$. The respondents' average age was 53.31 \pm 7.99 years; the urban areas inhabitants $(52.84 \pm 8.4)$ were slightly older than the inhabitants of rural areas $(52 \pm 8.05) \mathrm{p}=0.002$. Inhabitants of rural areas were less educated; they were more often in relationship, they smoked fewer cigarettes and more often declared physical activity $\geq 150$ minutes a week.

It was observed that MetS was significantly more frequent among inhabitants of rural areas $(40.6 \% ; n=810)$ compared to those living in the urban areas $(35.3 \%$; $\mathrm{n}=370$ ), $\mathrm{p}=0.005$. Similarly, in gender strata, MetS is more commonly diagnosed among males and females living in rural areas. Among the inhabitants of rural areas, the percentage of people with elevated FGB, HBP, elevated TGs levels and abnormal WC was significantly higher than in the respondents from the city.

\section{The Relationship Between MetS and Its Elements and the Place of Residence in the Gender Strata}

Table 2 presents the relationship between the place of residence and the presence of MetS and its elements in 


\begin{tabular}{|c|c|c|c|c|c|c|c|c|c|c|c|c|c|}
\hline \multirow{3}{*}{ 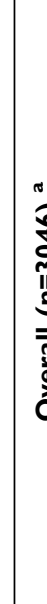 } & $a$ & & 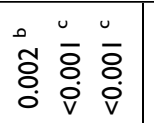 & & \multicolumn{3}{|l|}{$\overline{0}$} & \multirow[b]{2}{*}{ 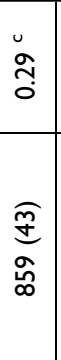 } & & \multirow{2}{*}{ 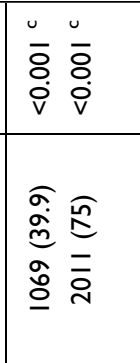 } & \multirow{2}{*}{ 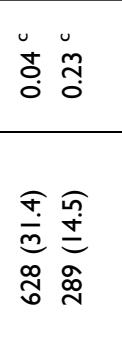 } & \multirow{2}{*}{ 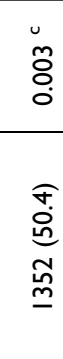 } & \multirow{2}{*}{ 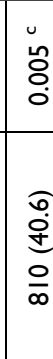 } \\
\hline & 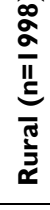 & & 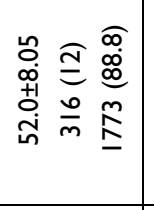 & & 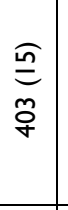 & 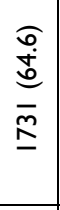 & $\begin{array}{l}\widehat{\sigma} \\
\stackrel{d}{d} \\
\text { fे }\end{array}$ & & & & & & \\
\hline & 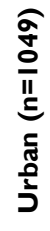 & & 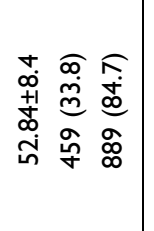 & & $\begin{array}{l}E \\
\bar{N}\end{array}$ & $\begin{array}{l}\text { E. } \\
\sigma \\
\bar{\sigma} \\
\bar{\infty}\end{array}$ & $\begin{array}{l}\widehat{m} \\
\stackrel{d}{d} \\
\frac{n}{m}\end{array}$ & $\begin{array}{l}\widehat{\bar{y}} \\
\stackrel{9}{y}\end{array}$ & & 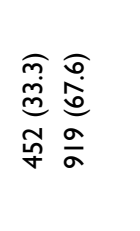 & 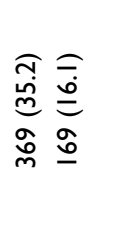 & $\begin{array}{l}\frac{\sigma}{\omega} \\
\frac{\infty}{9} \\
\frac{\infty}{6}\end{array}$ & 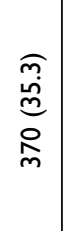 \\
\hline & $a$ & & 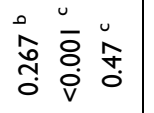 & & & ळे & & ữ & 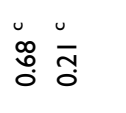 & ণ্ণ & un \\
\hline \multirow{2}{*}{ 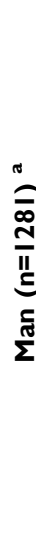 } & 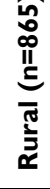 & & 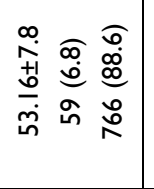 & & $\begin{array}{l}\widehat{m} \\
\stackrel{m}{d} \\
\mathbb{0} \\
\Phi\end{array}$ & $\begin{array}{l}\hat{\sigma} \\
\hat{\sigma} \\
o \\
o \\
\dot{y}\end{array}$ & 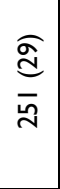 & $\begin{array}{l}\widehat{E} \\
\dot{y} \\
\frac{m}{\sigma}\end{array}$ & & 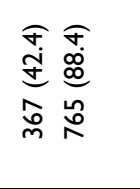 & 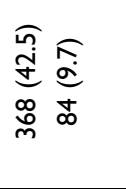 & 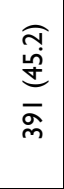 & 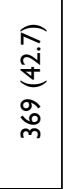 \\
\hline & 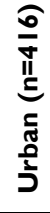 & & 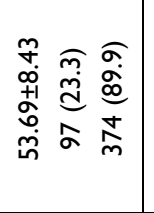 & & $\begin{array}{l}\widehat{\sigma} \\
\underset{d}{d} \\
\sim \alpha\end{array}$ & 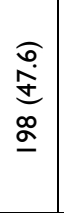 & 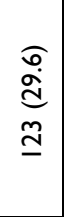 & 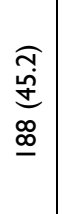 & & 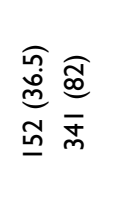 & 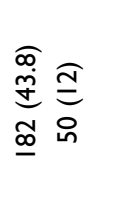 & $\begin{array}{l}\widehat{\overline{\vec{j}}} \\
\underline{\underline{\underline{I}}}\end{array}$ & 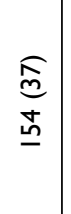 \\
\hline & $a$ & & 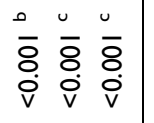 & & \multicolumn{3}{|l|}{$\begin{array}{l}u \\
\bar{o} \\
\dot{0}\end{array}$} & ח̋ & & 눙 & 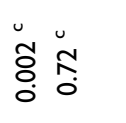 & $\begin{array}{l}0 \\
\vdots \\
0 \\
0\end{array}$ & ü \\
\hline & 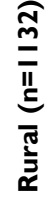 & & 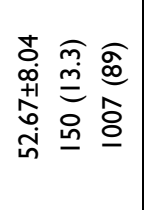 & & 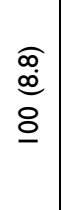 & 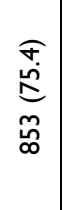 & 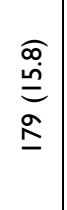 & 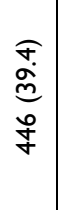 & & 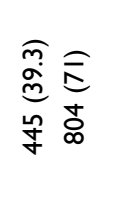 & 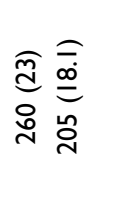 & 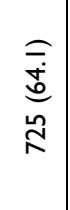 & $\begin{array}{l}\frac{\sigma}{\mathfrak{d}} \\
\bar{j}\end{array}$ \\
\hline & 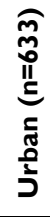 & & 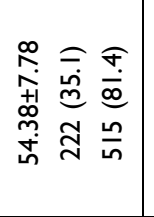 & & 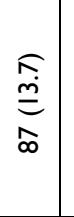 & 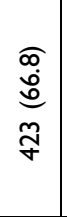 & 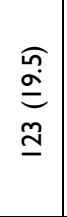 & 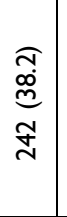 & & 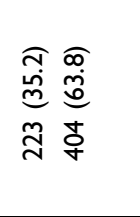 & 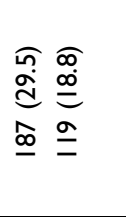 & 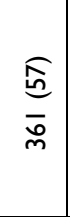 & $\begin{array}{l}\widehat{\widehat{\dot{p}}} \\
\stackrel{e}{\tilde{c}} \\
\frac{0}{N}\end{array}$ \\
\hline & \multicolumn{2}{|r|}{. } & 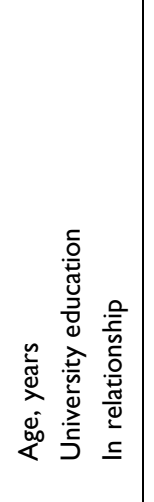 & 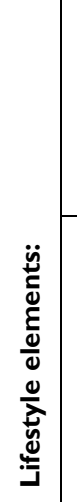 & 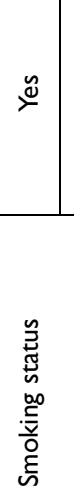 & zo & 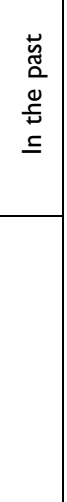 & 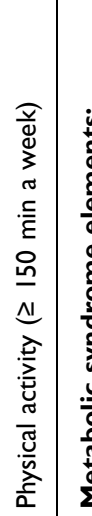 & 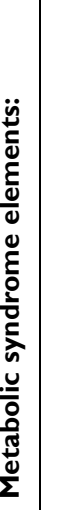 & 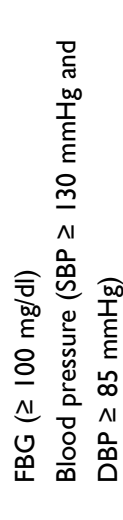 & 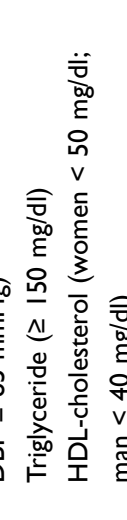 & 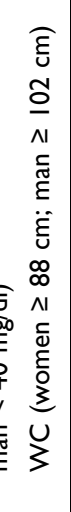 & 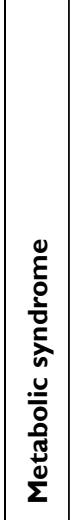 \\
\hline
\end{tabular}




\begin{tabular}{|c|c|c|c|c|c|c|c|c|c|c|c|c|c|}
\hline a & & 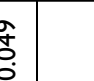 & $\bar{\delta}$ & $\stackrel{8}{\circ}$ & 慈 & & : & 弆 & & 莫: & ปั & ฐू๊ & 秀 \\
\hline 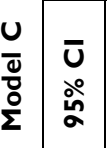 & & & 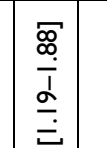 & 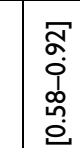 & 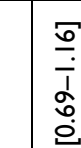 & & 离 & 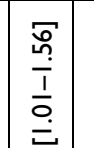 & & 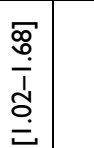 & 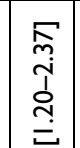 & 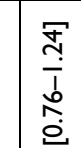 & 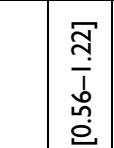 \\
\hline \% & -5 & $\stackrel{ \pm}{\stackrel{ \pm}{ \pm}}--$ & $\underset{\ddagger}{q}$ & $\stackrel{M}{0}$ & - & & \pm- & $\stackrel{\stackrel{a}{\Xi}}{ }$ & & $\overline{\underline{m}}$. & $\stackrel{\rho}{\underline{\underline{a}}}$ & Sa & - \\
\hline 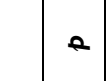 & & 草 & $\bar{\partial}$ & 高 & 浐 & & 彦 & 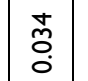 & & 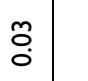 & : & : & 荾 \\
\hline
\end{tabular}

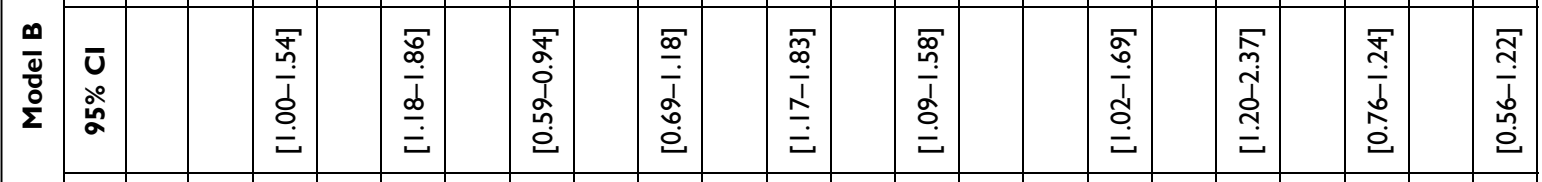

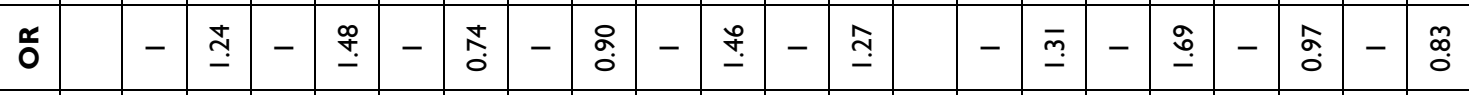

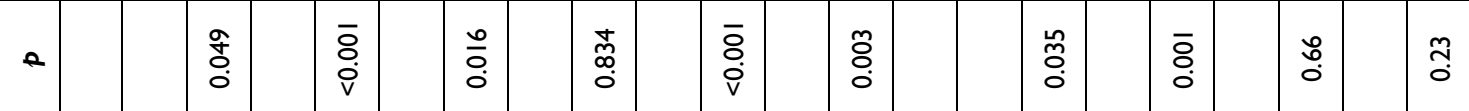

\begin{tabular}{|c|c|c|c|c|c|c|c|c|c|c|c|c|c|c|c|c|c|c|c|c|c|c|c|}
\hline \multirow[t]{2}{*}{ 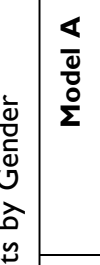 } & $\begin{array}{l}\bar{u} \\
\text { ㅇํํ } \\
\text { ڤू }\end{array}$ & & & 家 & & 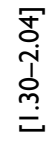 & & 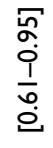 & & 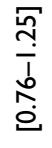 & & 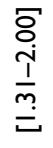 & & 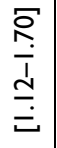 & & & | & & 恿 & & 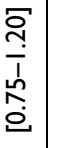 & & 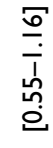 \\
\hline & ס̊ & & - & ָָ & - & $\stackrel{\bar{\sigma}}{ }$ & - & م̊ & - & ô & - & ֻِ & - & $\stackrel{\infty}{\underline{m}}$ & & - & $\stackrel{\stackrel{0}{m}}{\stackrel{9}{n}}$ & - & 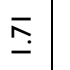 & - & 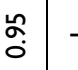 & & $\stackrel{\infty}{\infty}$ \\
\hline \multirow{3}{*}{ 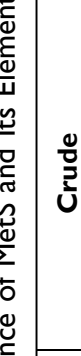 } & a & & & o. & & ర్ & & ฮั & & 尽 & & $\begin{array}{l}\text { to } \\
\text { o. }\end{array}$ & & 苂 & & & $\begin{array}{l}\text { 总 } \\
0 \\
0\end{array}$ & & o̊ & & $\begin{array}{l}\stackrel{\infty}{\circ} \\
:\end{array}$ & & $\bar{ָ}$ \\
\hline & $\begin{array}{l}\bar{u} \\
\text { ㅇํㅇ }\end{array}$ & & & 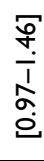 & & 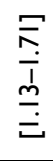 & & 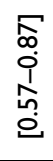 & & 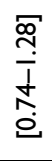 & & 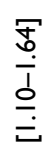 & & $\begin{array}{l}\frac{\bar{n}}{\underline{1}} \\
\frac{1}{\cos }\end{array}$ & & & 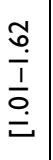 & & 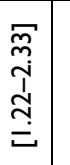 & & 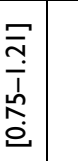 & & 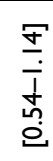 \\
\hline & ơ & & - & $\stackrel{\rho}{=}$ & - & $\stackrel{q}{q}$ & - & î. & - & $\stackrel{\circ}{\circ}$ & - & $\stackrel{\stackrel{m}{m}}{-}$ & - & $\stackrel{\widetilde{I}}{\underline{I}}$ & & - & ঙ্ : & - & 吕 & - & \begin{tabular}{c|c} 
o. \\
$\circ$
\end{tabular} & & $\hat{\hat{o}}$ \\
\hline \multirow[b]{2}{*}{ 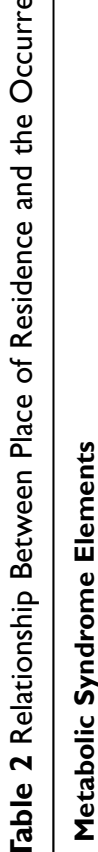 } & & & 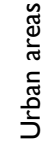 & 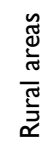 & 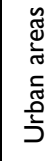 & 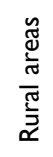 & 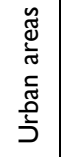 & 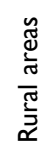 & 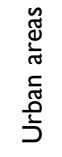 & 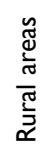 & 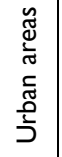 & 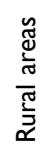 & 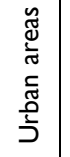 & 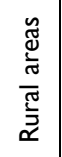 & & 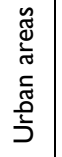 & 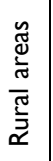 & 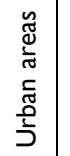 & 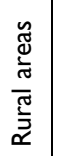 & 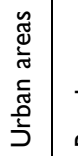 & 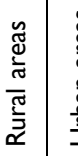 & & 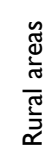 \\
\hline & 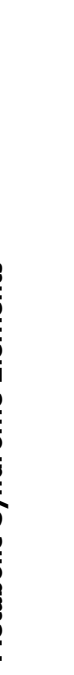 & 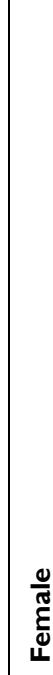 & 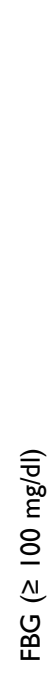 & & 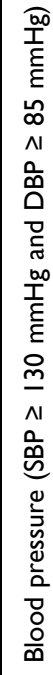 & & 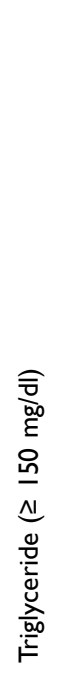 & & 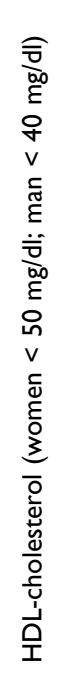 & & 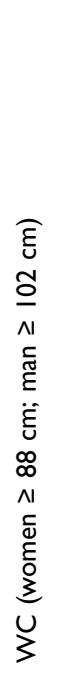 & & 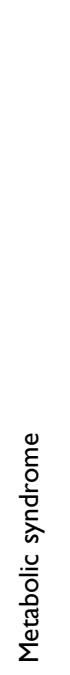 & & $\frac{\frac{0}{\pi}}{\frac{\pi}{\Sigma}}$ & 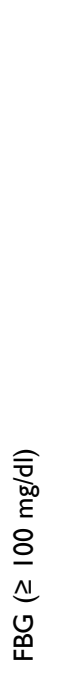 & & 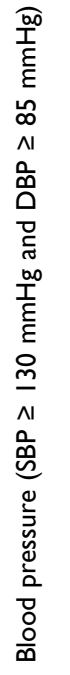 & & 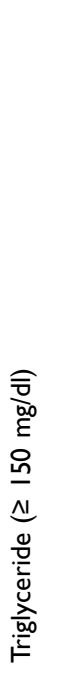 & 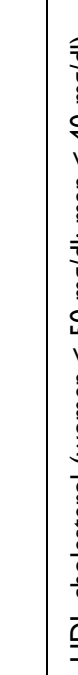 & 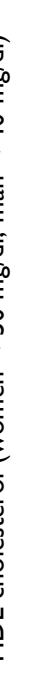 & \\
\hline
\end{tabular}




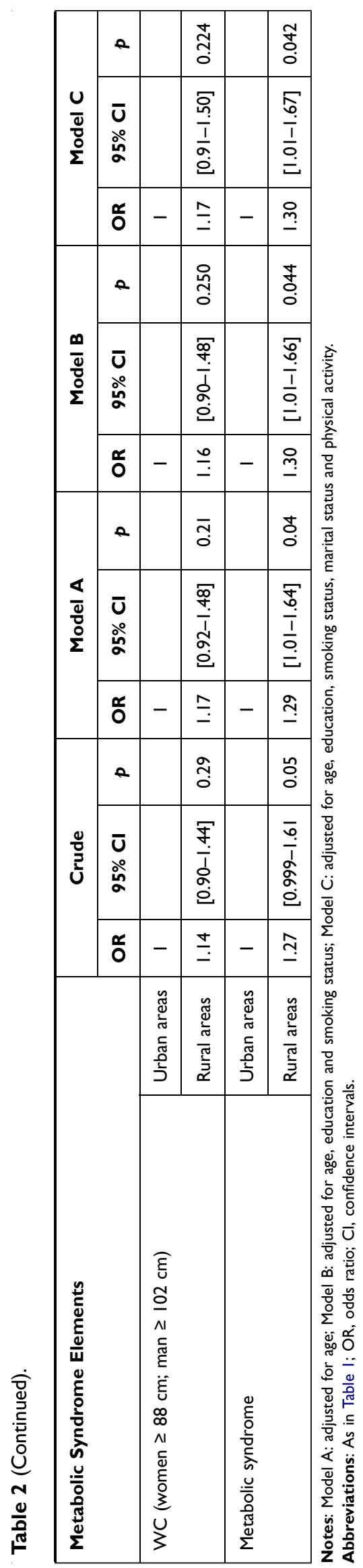

the gender strata. Adjusted for potential confounding variables (age, education, smoking, marital status, and level of physical activity) in both men and women, the odds of developing MetS were approximately 30\% higher in rural areas compared to urban residents (women: $\mathrm{OR}=1.25,95 \% \mathrm{CI}=1.01-1.56$; men: $\mathrm{OR}=1.30$, 95\% CI=1.01-1.67). Moreover, it was observed that the chance of HBP and FBG occurrence were significantly higher among the inhabitants of rural areas than the inhabitants of the city, both in women and men. The strongest relationship was found in men with HBP $\mathrm{OR}=1.69,95 \% \mathrm{CI}=1.20-2.37$. There was no relationship between the occurrence of low HDL-C and the place of residence in both gender groups.

A significant relationship was observed between the place of residence and the occurrence of increased levels of TGs and increased WC in women. In men, the direction of the relationship between the place of residence and the level of TGs and WC was similar, but it was not statistically significant.

\section{Discussion}

The aetiology of MetS is multifactorial; thus, environmental factors play an important role in triggering the cardiometabolic features of MetS. ${ }^{35,36}$ The incidence of MetS has increased in recent years, possibly due to changes in lifestyle, socioeconomic status (SES), and dietary habits. ${ }^{37}$ In our study, we found that after taking into account the potential confounding variables (age, education, smoking, marital status, and level of physical activity), both men and women residing in rural areas were characterized by an approximately $30 \%$ higher probability of developing MetS compared to urban residents. Among the elements of MetS, in women, all factors, except for the decreased HDL-C, were related to the place of residence. At the same time, in men, a significant relationship was observed between increased FGB and HBP and the residing areas.

$\mathrm{Xu}$ et $\mathrm{al}^{38}$ obtained similar results to our study. The authors assessed the prevalence of MetS in the urban, semi-urban, and rural population of China's Shaanxi Province. In the cited analysis of data from the China National Diabetes and Metabolic Disorders Study, conducted on a sample of 2446 people over the age of 20, it was found, correspondingly to our research, that the frequency of MetS was higher among the inhabitants of rural areas, especially among women. However, after implementing adjustments for confounding variables, the chance of MetS in the rural population were found to be $27.6 \%$ 
higher than in urban and peri-urban areas. Also, a higher incidence of MetS among inhabitants of rural areas was discovered in studies by Lee et $\mathrm{al}^{39}$ in the Korean population and by Trivedi et $\mathrm{al}^{16}$ in population of the United States However, the research investigating the prevalence of MetS in the Iranian population differs from the results of the presented study. Hossein-Ali et $\mathrm{al}^{40}$ and Jahangiry et $\mathrm{al}^{41}$ found that the incidence of MetS was greater in urban areas than in rural areas. Similarly, a greater prevalence of MetS among urban residents has been observed in the Nepalese population, ${ }^{42}$ that of South-West Nigeria ${ }^{43}$ and in Ecuador's ${ }^{44}$ and in a meta-analysis conducted in the Middle East as epidemiological studies by Ansarimoghaddam et al. $^{45}$ However, it is difficult to directly compare the results of our research with the above-cited studies' results. Enlisted literature uses different criteria for the diagnosis of MetSand includes populations demographically, socially, and culturally different and characterized by different SES.

A well-established predictor of MetS is SES, particularly as it regards educational attainment. ${ }^{46-49}$ A lower level of education is commonly regarded as a factor causing disparities in health. ${ }^{50}$ Higher education levels, in indirect ways-such as through economic welfare, healthrelated behaviour and mental resource-are believed to contribute to better health. ${ }^{51}$ Compared to those with lower education, people with higher education are more willing to look for employment and possess financial resources to purchase quality food or undertake regular physical activity, using sports and recreational infrastructure. Adults with higher education rarely engage in risky behaviours, such as smoking, and are more likely to engage in preventive behaviour. $^{52} \mathrm{~A}$ higher level of education also strengthens an individual's mental resources. For example, people with higher education have a greater number of social ties ${ }^{53}$ and experience depression less frequently. ${ }^{54}$ Moreover, a significant relationship has been found between gender and SES and MetS, ${ }^{55,56}$ although this relationship is not entirely clear due to conflicting results from studies conducted in different countries. ${ }^{55-61}$ Discrepancies in estimating the prevalence of MetS in rural and urban populations may be due to respondents' SES in each study. For example, people in rural areas are at greater risk of adverse health effects due to the overrepresentation of older adults, inferior education and low income. Our surveyed population, according to the data of the Central Statistical Office, Local Data Bank (GUS, BDL) for 2012 (data preceding our research), showed that many SES indicators of people living in the area of research were very unfavourable compared to the general population, ie, a higher percentage of respondents with primary education in relation to the general population $\left(27.55 \%\right.$ vs $23.2 \%$, respectively). ${ }^{62,63}$ In addition, the unemployment rate was $15.6 \%$ - the corresponding unemployment rate for the region is $15 \%$ while for the country the figure stood at $14 \%{ }^{64}$ The indicator concerning the number of people in families receiving social assistance for the Janowski district was relatively high - the social welfare system covered as much as $14.6 \%$ of the inhabitants (for comparison, the average in the Lubelskie Voivodeship is only 9.4\%). ${ }^{65}$ Among the risk factors potentially related to the development of MetS in the study group, the authors found that inhabitants of rural areas had a lower level of education compared to the surveyed city inhabitants.

Comparing the occurrence of risk factors included in MetS, we observed that the inhabitants of rural areas (both women and men) had a greater chance of developing HBP and an increased level of FGB. The results of our research correspond with the results of $\mathrm{Xu}$ et al. ${ }^{38}$ Our results and those cited above are consistent with other studies that found that the inhabitants of rural areas are more often diagnosed with $\mathrm{HBP}^{66,67}$ and more often have an elevated FGB level ${ }^{68,69}$ compared to the inhabitants of urban areas. That characteristic of rural areas can be explained by the fact that progressive urbanization brings both higher incomes and unhealthy lifestyles to the inhabitants of rural areas, as poor socioeconomic conditions in early life increase the risk of obesity later on when accomplishing an economically improved life. ${ }^{70}$ Along with the transformation of farms, small farms, often passed down from generation to generation, which have become unprofitable to run, resulting in a lack of work on the farmland. Importantly, this deficit in farm-related physical activity and energy expenditure will never be compensated through recreational physical activity. ${ }^{71}$ Moreover, the rapid urbanization of rural areas changed its inhabitants' diet; increased the supply of high-energy food and lead to the increased consumption of animal products, associated with a greater supply of animal fats in the diet. ${ }^{72}$ Obesity and metabolic have become the direct consequences of reduced physical activity and inadequate diet among the inhabitants of rural areas, as shown in our study. In increasingly developing rural areas, the environmental risk factors such as air and water pollution in urban areas affecting the disease have become, to some extent, more 
frequent. Rural residents do not take advantage of the available opportunities such as sport and recreational infrastructure, better medical insurance and the easier access to health care to improve their health. For this reason, the subjects of our study were more likely to have MetS, despite the exclusion of socio-demographic and lifestyle risk factors, indicating that our excluded factors only partially explain the greater chance of MetS among rural areas' residents.

In our study, an increased number of MetS elements was significantly related to the place of residence, especially in women compared to men. The incidence of MetS risk factors varies with gender, race, ethnicity, and age. While some authors suggest the advantages of specific risk factors in predicting all-cause mortality in some populations, ${ }^{73}$ this remains to be explored in rural and urban populations.

There is a visible increase in recognition concerning the environmental pollution adversely affecting the development of MetS. The development of modern farming practices has been essential to increasing food supplies and play a key role as food demand rises globally. Also, endocrine-disrupting chemicals (EDC) play a key role in the development of metabolic diseases. Those substances are defined as

an exogenous substance or mixture that alters the function (s) of the endocrine system and consequently causes adverse effects in intact organism, or its progeny or (sub) population. ${ }^{74}$

Agricultural insecticides, herbicides, and fertilizers have improved the stability of the food supply chain; nonetheless, by contaminating rural environments with these substances ${ }^{75}$ they may also contribute to the increase in MetS. ${ }^{76}$ Exposure to chemicals in the environment positively correlates with the increase in the development of metabolic diseases. ${ }^{77-79}$ Among the chemical substances present in the environment, phytates, ${ }^{80}$ organophosphate esters, ${ }^{81}$ perfluoroalkyl substances ${ }^{82}$ or phenols and parabens, inter alia, increase the likelihood of MetS occurrence. ${ }^{83}$ Heavy metals may also play an essential role in the development of not only cardiovascular complications $^{84}$ but also MetS, which due to the development of industry, traffic, smoking or changes in food production technology, constitute an imperative element of environmental pollution, also among residents of rural areas. ${ }^{85}$ Heavy metals influencing the higher risk of metabolic syndrome turned out to be, among others, mercury, ${ }^{86}$ arsenic, cadmium, lead, ${ }^{87}$ copper and zinc. ${ }^{88}$ The last important piece of the puzzle of environmental factors that may potentially cause exposition to the more frequent occurrence of MetS is air pollution, as demonstrated in the research by, for example, Zhang et al, ${ }^{89}$ Yang et al ${ }^{90}$ or Matthiessen et al. ${ }^{91} \mathrm{MetS}$ is associated with the development of many other diseases. Available epidemiological and laboratory data indicate that MetS is a potential risk factor for developing certain types of cancers ${ }^{92}$ such as colorectal, liver, pancreatic and postmenopausal breast cancer. ${ }^{93}$ It has been postulated that the link between MetS and cancer is due to obesity, high blood glucose and other components of MetS. ${ }^{94}$ Some studies suggest that high blood pressure and dyslipidaemia are associated with a higher risk of developing cancer. ${ }^{95,96}$ Investigating the relationship between MetS and cancer is challenging because MetS is a clinical entity involving multiple cardiometabolic risk factors, and therefore the effects of a single component of MetS on cancer development and progression may overlap with others resulting in neutral, additive, or synergistic effects. These limits can be partially overcome by assessing the importance of each MetS component in terms of both the risk and prognosis of certain cancers and then comparing them to those induced by all MetS components. ${ }^{97}$ Determining which MetS component influences its association with cancer risk is vital as preventive and treatment interventions may benefit MetSrelated cancers and cancer mortality. ${ }^{98}$ However, while overweight/obesity are some causes of cancer development and mortality, which are preventable ${ }^{99,100}$ the relationship between the other components of MetS (hyperglycemia, dyslipidemia, hypertension) and cancer is still under discussion. ${ }^{101,102}$

Despite the growing number of initiatives implemented to improve society's health and well-being, we still face an extensive number of health and socioeconomic issues and polarization of society through increasing health and socioeconomic inequalities at the local, national, and international levels. Mitigating inequalities in the health condition of the inhabitants of Central and Eastern Europe, as with the example of the Janów Lubelski district analysed in our research, is an essential priority in advancing the goal of sustainable development for Poland and the European Union. ${ }^{103}$ Given that the incidence of MetS has been increasing in recent years, ${ }^{104,105}$ identifying a population at risk of MetS would be associated with a more effective screening policy and with prevention too (primary and secondary). Our research showed that 
people in rural areas were more likely to develop MetS Therefore, the likelihood of developing medical complications such as diabetes (DM) and CVD in this population was higher. Comprehensive community programs designed to combat MetS and improve health and quality of life through various approaches, that include education, prevention, screening and treatment, are all needed in rural areas. ${ }^{106}$ Such programs supported by community leaders and local institutions, including schools, health care facilities and workplaces, would ensure that nearly all local community members participate, reducing the risk of MetS and its complications. Second, such a plan should include the modernization of the living environment in rural areas so that it is conducive to active recreation, ie, the construction of sidewalks, parks, exercise facilities, sports fields, bicycle paths, etc. Finally, an important element in the prevention of MetS is promoting healthy nutrition by educating people about the health effects of certain substances contained in food products, subsidizing healthy food and increasing the availability of healthy food markets from local producers. The implementation by local governments of preventive health policy programs, in close cooperation with the primary care physician, should be one of the essential elements of an efficient and effective health care system which prioritizes preventive measures in the MetS area, especially in regions with a high exposure. ${ }^{107}$ Their task would be to facilitate the use of preventive services such as screening and counselling patients about healthy lifestyles. ${ }^{108}$

\section{The Strength of the Study}

It is one of the few cross-sectional studies in Poland conducted with a large sample. Moreover, the study was conducted among the population affected by one of the lowest HDI indices out of all districts in Poland. The analysis also shed new light on the prevalence of metabolic syndrome and its associated risk factors.

\section{The Limitations of the Study}

Our study has some limitations that should be considered. First, the study covered a population from the southeastern region of Poland. Moreover, the respondents' selection did not take into account the procedure of randomization and stratification of the sample by age and gender. Second, this study's cross-sectional design and the conducted analysis of the results limit its power to causeand-effect inference; it only shows a certain tendency of relationships. Third, the study included people with a medium and high cardiovascular risk on the SCORE scale. Therefore, the results obtained cannot be generalized to the entire population. The fourth limitation is the physical activity questionnaire. In terms of physical activity, the respondents were asked only one question.Finally, the fifth limitation of our research is the lack of analyses of the profession's impact on the risk of MetS. As the studies by Strauß et al ${ }^{109}$ show, the occupation significantly influences the risk of MetS. The authors of the cited studies indicate that in the group of office workers they studied, compared to firefighters, they observed abnormal levels of HDL, TG, BMI, BP and WC, but also MetS was more often diagnosed in office workers whose work was associated with a sedentary lifestyle than in firefighters.

\section{Conclusion \& Recommendations Conclusion}

The more frequent occurrence of MetS was observed among respondents living in rural areas compared to those living in urban areas. Similarly, with gender strata, MetS is more commonly diagnosed among males and females living in rural areas. Among the elements constituting MetS, a more frequent occurrence of elevated FGB and HBP was observed among inhabitants of rural areas, both in women and men. There was no relationship between the place of residence and the decreased HDL-C concentration. The association between MetS and elevated TGs and abnormal WC was found in women. The abovementioned differences occurred regardless of the respondents' age, education, smoking and physical activity.

\section{Recommendations}

\section{Health Policy Makers}

Policymakers should design and raise funds for health programs dedicated to small rural local communities in order to eliminate factors that increase the risk of MetS. A legal, organizational, and financial framework should be developed to formally involve local rural communities in the implementation of such programs.

\section{For Health Professionals}

Healthcare workers, especially in rural areas, should engage in the process of education, prevention and promotion as regards a healthy lifestyle through a holistic approach to patients via coordinated health activities. Future Mets prevention and education programs should be targeted at the primary care level, primarily at people from rural backgrounds, both adult women and men. 


\section{For Researchers}

Future research projects should include long-term MetS cohorts to better understand the critical determinants of the development of this premorbid state, including factors related to insulin resistance, poor diet, lack of physical activity, obesity and genetic burden. Moreover, further research should take into account the search for barriers and for factors eliminating barriers to preventive services that are offered by the health care system among the inhabitants of rural areas.

\section{For the Community}

There is a need for wider formal involvement of community members in managing the practice of Individual Health Competency in the area of early detection and correction of MetS-related disorders.

\section{Abbreviations}

MetS, metabolic syndrome; WC, waist circumference; EDC, endocrine-disrupting chemicals; FBG, fasting plasma glucose; HBP, high blood pressure; TGs, triglycerides; HDL-C, high-density lipoprotein cholesterol; T2DM, type 2 diabetes; CVD, cardiovascular disease; WOBASZ, Multicentre National Population Health Examination Survey; AHA, American Heart Association; NHLBI, National Heart, Lung, and Blood Institute; IDF, International Diabetes Federation; PURE, Prospective Urban and Rural Epidemiological Study; PONS, Polish Norwegian Study; SCORE, Systematic COronary Risk Evaluation; SMRs, standardized mortality ratios; HDI, human development index; BP, blood pressure; SBP, systolic blood pressure; DBP, diastolic blood pressure; NCEP ATP III, National Cholesterol Education Program - Third Adult Treatment Panel; OR, odds ratio; CI, confidence intervals; SES, socioeconomic status; GUS, BDL, The Central Statistical Office, The Local Data Bank; DM, diabetes mellitus.

\section{Data Sharing Statement}

Data are available by contacting the corresponding author.

\section{Ethics Approval}

This study was approved by the Bioethical Commission of the Medical University in Lublin (no. KE-0254/112/2014) and was conducted in accordance with the Declaration of Helsinki. All the final participants provided written informed consent.

\section{Acknowledgments}

The authors would like to express our gratitude to Maciej Polak, DSc for his assistance in preparing statistical analyses.

\section{Funding}

The research was performed as part of the project entitled "Follow Your Heart-prevention and health promotion program for cardiovascular diseases in the Janów Lubelski district" financed by the Norwegian Financial Mechanism 2009-2014 under the PL Program 13 "Limiting social inequities in health" and the state budget. Statistical analysis of the material, translations, and publication costs were financed from the own resources of the Medical University of Lublin as part of the statutory activity in the area of maintaining research potential (MNmb 615 and DS 519).

\section{Disclosure}

The authors declare no conflicts of interest in this work.

\section{References}

1. Huang PL. A comprehensive definition for metabolic syndrome. Dis Model Mech. 2009;2(5-6):231-237. doi:10.1242/ dmm.001180

2. Galassi A, Reynolds K, He J. Metabolic syndrome and risk of cardiovascular disease: a meta-analysis. Am J Med. 2006;119 (10):812-819. doi:10.1016/j.amjmed.2006.02.031

3. Ahluwalia N, Andreeva VA, Kesse-Guyot E, Hercberg S. Dietary patterns, inflammation and the metabolic syndrome. Diabetes Metab. 2013;39(2):99-110. doi:10.1016/j.diabet.2012.08.007

4. Gathirua-Mwangi WG, Monahan PO, Murage MJ, Zhang J. Metabolic syndrome and total cancer mortality in the Third National Health and Nutrition Examination Survey. Cancer Causes Control. 2017;28(2):127-136. doi:10.1007/s10552-0160843-1

5. Roomi MA, Mohammadnezhad M. Prevalence of metabolic syndrome among apparently healthy workforce. J Ayub Med Coll Abbottabad. 2019;31(2):252-254.

6. Sygnowska E, Piwońska A, Waśkiewicz A, Broda G. Socioeconomic factors and the risk of metabolic syndrome in the adult Polish population: the WOBASZ study. Kardiol Pol. 2012;70(7):718-727.

7. Czekajło A, Różańska D, Zatońska K, Szuba A, Regulska-Ilow B. Association between dietary patterns and metabolic syndrome in the selected population of Polish adults-results of the PURE Poland Study. Eur J Public Health. 2019;29(2):335-340. doi:10.1093/eurpub/cky207

8. Janszky I, Vatten L, Romundstad P, et al. Metabolic syndrome in Poland - the PONS Study. Ann Agric Environ Med. 2011;18 (2):270-272.

9. Kassi E, Pervanidou P, Kaltsas G, Chrousos G. Metabolic syndrome: definitions and controversies. BMC Med. 2011;9(1):48. doi:10.1186/1741-7015-9-48

10. Cornier MA, Dabelea D, Hernandez TL, et al. The metabolic syndrome. Endocr Rev. 2008;29(7):777-822. doi:10.1210/ er.2008-0024 
11. Dalal S, Beunza JJ, Volmink J, et al. Non-communicable diseases in sub-Saharan Africa: what we know now. Int $J$ Epidemiol. 2011;40(4):885-901. doi:10.1093/ije/dyr050

12. Prabhakaran D, Chaturvedi V, Shah P, et al. Differences in the prevalence of metabolic syndrome in urban and rural India: a problem of urbanization. Chronic Illn. 2007;3(1):8-19. doi:10.1177/1742395307079197

13. Gu D, Reynolds K, Wu X, et al. Prevalence of the metabolic syndrome and overweight among adults in China. Lancet. 2005;365(9468):1398-1405. doi:10.1016/S0140-6736(05)66375-1

14. Mohamud WN, Ismail AA, Sharifuddin A, et al. Prevalence of metabolic syndrome and its risk factors in adult Malaysians: results of a nationwide survey. Diabetes Res Clin Pract. 2011;91(2):239-245. doi:10.1016/j.diabres.2010.11.025

15. Jaspers Faijer-Westerink H, Kengne AP, Meeks KAC, Agyemang C. Prevalence of metabolic syndrome in sub-Saharan Africa: a systematic review and meta-analysis. Nutr Metab Cardiovasc Dis. 2020;30(4):547-565. doi:10.1016/j. numecd.2019.12.012

16. Trivedi T, Liu J, Probst JC, Martin AB. The metabolic syndrome: are rural residents at increased risk? J Rural Health. 2013;29 (2):188-197. doi:10.1111/j.1748-0361.2012.00422.x

17. Lim S, Jang HC, Lee HK, Kimm KC, Park C, Cho NH. A rural-urban comparison of the characteristics of the metabolic syndrome by gender in Korea: the Korean Health and Genome Study (KHGS). J Endocrinol Investig. 2006;29(4):313-319. doi:10.1007/BF03344102

18. van Zyl S, van der Merwe LJ, Walsh CM, Groenewald AJ, Rooyen FC. Risk-factor profiles for chronic diseases of lifestyle and metabolic syndrome in an urban and rural setting in South Africa. Afr J Prim Health Care Fam Med. 2012;4(1):346. doi:10.4102/phcfm.v4i1.346

19. Khang YH. Different longevity by village. In: Lee CG, editor. Chasing Health Inequality in Korea: A Report for Making It Social Issue. Seoul: Publishing Company Meem; 2007:67-79.

20. Yang BY, Liu KK, Markevych I, et al. Association between residential greenness and metabolic syndrome in Chinese adults. Environ Int. 2020;135:105388. doi:10.1016/j.envint.2019.105388

21. Program PL 13. Publication of mortality rates for selected districts. Available from: http://archiwum.zdrowie.gov.pl/aktual nosc-27-2136-

Program_PL_13_publikacja_wskaznikow_umieralnosci_dla_ wybranych powiatow.html. Accessed October 19, 2018.

22. Arak P, Ivanov A, Peleah M, et al. Krajowy Raport o Rozwoju Spolecznym Polska 2012. Poland: Rozwój regionalny i lokalny. [National report on social development of Poland 2012. Regional and local development] Warsaw: United Nations Development Programme; 2012.

23. Central Statistical Office, Local Data Bank (GUS, BDL). Deaths by causes for districts in 2012. Available from: https://bdl.stat. gov.pl/BDL/dane/podgrup/temat/3/534/2286. Accessed October 19, 2018

24. Nolan PB, Carrick-Ranson G, Stinear JW, Reading SA, Dalleck LC. Prevalence of metabolic syndrome and metabolic syndrome components in young adults: a pooled analysis. Prev Med Rep. 2017;7:211-215. doi:10.1016/j.pmedr.2017.07.004

25. Zdrojewski T, Jankowski P, Bandosz P, et al. A new version of cardiovascular risk assessment system and risk charts calibrated for Polish population. Kardiol Pol. 2015;73(10):958-961. doi:10.5603/KP.2015.0182

26. Lloyd-Jones DM, Hong Y, Labarthe D, et al. Defining and setting national goals for cardiovascular health promotion and disease reduction: the American Heart Association's strategic Impact Goal through 2020 and beyond. Circulation. 2010;121 (4):586-613. doi:10.1161/CIRCULATIONAHA.109.192703
27. WHO. Waist Circumference and Waist-Hip Ratio: Report of a WHO Expert Consultation. Geneva: WHO Library Cataloguingin; 2008.

28. Weber MA, Schiffrin EL, White WB, et al. Clinical practice guidelines for the management of hypertension in the community: a statement by the American Society of Hypertension and the International Society of Hypertension. J Clin Hypertens. 2014;16 (1):14-26. doi:10.1111/jch.12237

29. Cleeman JI, Grundy SM, Becker D, Clark L. Expert panel on detection, evaluation, and treatment of high blood cholesterol in adults. Executive summary of the third report of The National Cholesterol Education Program (NCEP) expert panel on detection, evaluation, and treatment of high blood cholesterol in adults (Adult Treatment Panel III). JAMA. 2001;285(19):2486-2497. doi:10.1001/jama.285.19.2486

30. Nilsson PM, Engström G, Hedblad B. The metabolic syndrome and incidence of cardiovascular disease in non-diabetic subjects-a population-based study comparing three different definitions. Diabet Med. 2007;24(5):464-472. doi:10.1111/j.14645491.2007.02142.x

31. Alberti KG, Zimmet P, Shaw J. IDF Epidemiology Task Force Consensus Group. The metabolic syndrome-a new worldwide definition. Lancet. 2005;366(9491):1059-1062. doi:10.1016/ S0140-6736(05)67402-8

32. Pouliot MC, Després JP, Lemieux S, et al. Waist circumference and abdominal sagittal diameter: best simple anthropometric indexes of abdominal visceral adipose tissue accumulation and related cardiovascular risk in men and women. Am J Cardiol. 1994;73(7):460-468. doi:10.1016/0002-9149(94)90676-9

33. Athyros VG, Ganotakis ES, Tziomalos K, et al. Comparison of four definitions of the metabolic syndrome in a Greek (Mediterranean) population. Curr Med Res Opin. 2010;26 (3):713-719. doi:10.1185/03007991003590597

34. Chrostowska M, Szyndler A, Polonis K, Tykarski A, Narkiewicz K. The abdominal obesity prevalence among hypertensives patients in Poland - the consequence of diagnostic criteria change in new Polish Society of Hypertension 2011 guidelines. Arterial Hypertension. 2011;15(3):155-162.

35. Brown AE, Walker M. Genetics of insulin resistance and the metabolic syndrome. Curr Cardiol Rep. 2016;18(8):75. doi:10.1007/s11886-016-0755-4

36. Elder SJ, Lichtenstein AH, Pittas AG, et al. Genetic and environmental influences on factors associated with cardiovascular disease and the metabolic syndrome. J Lipid Res. 2009;50 (9):1917-1926. doi:10.1194/j1r.P900033-JLR200

37. Salas R, BibiloniMdel M, Ramos E, et al. Metabolic syndrome prevalence among Northern Mexican adult population. PLoS One. 2014;9(8):e105581. doi:10.1371/journal.pone.0105581

38. Xu S, Ming J, Yang $\mathrm{C}$, et al. Urban, semi-urban and rural difference in the prevalence of metabolic syndrome in Shaanxi province, northwestern China: a population-based survey. $B M C$ Public Health. 2014;14:104. doi:10.1186/1471-2458-14-104

39. Lee S, Shin Y, Kim Y. Risk of metabolic syndrome among middle-aged Koreans from rural and urban areas. Nutrients. 2018;10(7):859. doi:10.3390/nu10070859

40. Hossein-Ali N, Abbas R, Mozhgan S, Haleh G. Prevalence of metabolic syndrome and its components among a population-based study in south of Iran, PERSIAN Kharameh cohort study. Clin Epidemiol Global Health. 2020;8(3):678-683. doi:10.1016/j.cegh.2020.01.001

41. Jahangiry L, Khosravi-Far L, Sarbakhsh P, Kousha A, EntezarMahdi R, Ponnet K. Prevalence of metabolic syndrome and its determinants among Iranian adults: evidence of IraPEN survey on a bi-ethnic population. Sci Rep. 2019;9(1):7937. doi:10.1038/s41598-019-44486-8 
42. Mehata S, Shrestha N, Mehta RK, Bista B, Pandey AR, Mishra SR. Prevalence of the metabolic syndrome and its determinants among Nepalese adults: findings from a nationally representative cross-sectional study. Sci Rep. 2018;8(1):14995. doi:10.1038/s41598-018-33177-5

43. Adejumo E, Ogundahunsi O, Adejumo O, Sotunsa J, Jagun O. Prevalence of metabolic syndrome in a rural and urban community in South-West Nigeria using three different definitions. Int $J$ Trop Dis Health. 2017;24(2):1-9. doi:10.9734/IJTDH/2017/33993

44. Orces CH, Gavilanez EL. The prevalence of metabolic syndrome among older adults in Ecuador: results of the SABE survey. Diabetes Metab Syndr. 2017;11(suppl 2):S555-S560. doi:10.1016/j.dsx.2017.04.004

45. Ansarimoghaddam A, Adineh HA, Zareban I, Iranpour S, HosseinZadeh A, Kh F. Prevalence of metabolic syndrome in middle-east countries: meta-analysis of cross-sectional studies. Diabetes Metab Syndr. 2018;12(2):195-201. doi:10.1016/j. dsx.2017.11.004

46. Dallongeville J, Cottel D, Ferrières J, et al. Household income is associated with the risk of metabolic syndrome in a sex-specific manner. Diabetes Care. 2005;28(2):409-415. doi:10.2337/ diacare.28.2.409

47. Silventoinen K, Pankow J, Jousilahti P, Hu G, Tuomilehto J. Educational inequalities in the metabolic syndrome and coronary heart disease among middle-aged men and women. Int J Epidemiol. 2005;34(2):327-334. doi:10.1093/ije/dyi007

48. Lucove JC, Kaufman JS, James SA. Association between adult and childhood socioeconomic status and prevalence of the metabolic syndrome in African Americans: the Pitt County Study. Am J Public Health. 2007;97(2):234-236. doi:10.2105/AJPH.2006.087429

49. Wu HF, Tam T, Jin L, et al. Age, gender, and socioeconomic gradients in metabolic syndrome: biomarker evidence from a large sample in Taiwan, 2005-2013. Ann Epidemiol. 2017;27 (5):315-322. doi:10.1016/j.annepidem.2017.04.003

50. Link BG, Phelan J. Social conditions as fundamental causes of disease. J Health Soc Behav. 1995;35:80-94. doi:10.2307/ 2626958

51. Mirowsky J, Ross CE. Education, Social Status, and Health. New York: Aldine de Gruyter; 2003.

52. Monte LM, Ellis RR. Fertility of Women in the United States: 2012. Current Population Reports. Washington DC: U.S. Census Bureau; 2014.

53. Miech RA, Shanahan MJ. Socioeconomic status and depression over the life course. J H Soc Behav. 2000;41(2):162-176. doi: $10.2307 / 2676303$

54. Rosenberg-Lee M, Barth M, Menon V. What difference does a year of schooling make? Maturation of brain response and connectivity between 2 nd and 3rd grades during arithmetic problem solving. Neuroimage. 2011;57(3):796-808. doi:10.1016/j. neuroimage.2011.05.013

55. Santos AC, Ebrahim S, Barros H. Gender, socio-economic status and metabolic syndrome in middle-aged and old adults. $B M C$ Public Health. 2008;8(1):62. doi:10.1186/1471-2458-8-62

56. Al-Daghri NM, Alkharfy KM, Al-Attas OS, et al. Genderdependent associations between socioeconomic status and metabolic syndrome: a cross-sectional study in the adult Saudi population. BMC Cardiovascular Disord. 2014;14:51. doi:10.1186/1471-2261-14-51

57. Buckland G, Salas-Salvadó J, Roure E, Bulló M, Serra-Majem L. Sociodemographic risk factors associated with metabolic syndrome in a Mediterranean population. Public Health Nutr. 2008;11(12):1372-1378. doi:10.1017/S1368980008003492

58. Pan A, Keum N, Okereke OI, et al. Bidirectional association between depression and metabolic syndrome: a systematic review and meta-analysis of epidemiological studies. Diabetes Care. 2012;35(5):1171-1180. doi:10.2337/dc11-2055
59. Vernay M, Salanave B, de Peretti C, et al. Metabolic syndrome and socioeconomic status in France: the French Nutrition and Health Survey (ENNS, 2006-2007). Int J Public Health. 2013;58(6):855-864. doi:10.1007/s00038-013-0501-2

60. Loucks EB, Rehkopf DH, Thurston RC, Kawachi I. Socioeconomic disparities in metabolic syndrome differ by gender: evidence from NHANES III. Ann Epidemiol. 2007;17 (1):19-26. doi:10.1016/j.annepidem.2006.07.002

61. Hosseinpour-Niazi S, Mirmiran P, Hosseinpanah F, FallahGhohroudy A, Azizi F. Association of marital status and marital transition with metabolic syndrome: tehran lipid and glucose study. Int J Endocrinol Metab. 2014;12(4):e18980. doi:10.5812/ijem. 18980

62. Strzelecki Z, Szymborski J Zachorowalność i umieralność na choroby układu krążenia a sytuacja demograficzna Polski. [Morbidity and mortality of cardiovascular diseases and the demographic situation of Poland]. Available from: https://bip.stat.gov.pl/files/gfx/bip/pl/ zamowieniapubliczne/426/248/1/81_gp_rrl_2015_monografia_kar diologiczna.pdf. Accessed October 19, 2018.

63. Central Statistical Office. Report on results. National census of population and apartments 2011. Available from: https://stat.gov. pl/cps/rde/xbcr/gus/lud_raport_z_wynikow_NSP2011.pdf. Accessed October 19, 2018.

64. Statistical Office in Lublin. Registered unemployment in Lubelskie Voivodship in 2014. Available from: http://wuplublin. praca.gov.pl/-/1355901-sytuacja-na-rynku-pracy-w-wojewodzt wie-lubelskim-luty-2015. Accessed October 19, 2018.

65. Central Statistical Office. Beneficiaries of social assistance and family benefits in 2012. Available from: https://stat.gov.pl/files/ gfx/portalinformacyjny/pl/defaultaktualnosci/5487/6/3/5/benefic jenci_pomocy_spolecznej_i_swiadczen_rodzinnych_2012.pdf. Accessed October 19, 2018.

66. Wang J, Sun W, Wells GA, et al. Differences in prevalence of hypertension and associated risk factors in urban and rural residents of the northeastern region of the People's Republic of China: a cross-sectional study. PLoS One. 2018;13(4):e0195340. doi:10.1371/journal.pone. 0195340

67. Nuotio J, Vähämurto L, Pahkala K, et al. CVD risk factors and surrogate markers - urban-rural differences. Scand J Public Health. 2020;48(7):752-761. doi:10.1177/1403494819869816

68. Zatońska K, Połtyn-Zaradna K, Einhorn J, et al. Differences in prevalence of diabetes mellitus type 2 and impaired fasting glucose between urban and rural areas according to PURE Poland substudy. Int $J$ Diabetes Dev Ctries. 2017;37:305-312. doi:10.1007/s13410-016-0523-4

69. Aung WP, Htet AS, Bjertness E, Stigum H, Chongsuvivatwong V, Kjøllesdal MKR. Urban-rural differences in the prevalence of diabetes mellitus among 25-74 year-old adults of the Yangon Region, Myanmar: two cross-sectional studies. BMJ Open. 2018;8(3):e020406. doi:10.1136/bmjopen-2017-020406

70. Kavikondala S, Schooling CM, Jiang CQ, et al. Pathways to obesity in a developing population: the Guangzhou Biobank Cohort Study. Int J Epidemiol. 2009;38(1):72-82. doi:10.1093/ ije/dyn221

71. Haase A, Steptoe A, Sallis JF, Wardle J. Leisure-time physical activity in university students from 23 countries: associations with health beliefs, risk awareness, and national economic development. Prev Med. 2004;39(1):182-190. doi:10.1016/j. ypmed.2004.01.028

72. Popkin BM, Du S. Dynamics of the nutrition transition toward the animal foods sector in China and its implications: a worried perspective. $J$ Nutr. $2003 ; 133$ (11Suppl 2):3898S-3906S. doi:10.1093/jn/133.11.3898S

73. Franklin SS, Gustin W, Wong ND, et al. Hemodynamic patterns of age-related changes in blood pressure. The Framingham Heart Study. Circulation. 1997;96(1):308-315. doi:10.1161/01. cir.96.1.308 
74. Bergman A, Heindel JJ, Kasten T, et al. The impact of endocrine disruption: a consensus statement on the state of the science. Environ Health Perspect. 2013;121(4):A104-A106. doi:10.1289/ ehp. 1205448

75. Sărmăşan C, Drăghici S, Dain L. Identification, communication and management of risks relating to drinking water pollution in Bihor County. Environ Eng Manage J. 2008;7(6):769-774. doi:10.30638/eemj.2008.102

76. De Long NE, Holloway AC. Early-life chemical exposures and risk of metabolic syndrome. Diabetes Metab Syndr Obes. 2017;10:101-109. doi:10.2147/DMSO.S95296

77. Casals-Casas C, Desvergne B. Endocrine disruptors: from endocrine to metabolic disruption. Annu Rev Physiol. 2011;73:135-162. doi:10.1146/annurev-physiol-012110-142200

78. Baillie-Hamilton PF. Chemical toxins: a hypothesis to explain the global obesity epidemic. J Altern Complement Med. 2002;8 (2):185-192. doi:10.1089/107555302317371479

79. Neel BA, Sargis RM. The paradox of progress: environmental disruption of metabolism and the diabetes epidemic. Diabetes. 2011;60(7):1838-1848. doi:10.2337/db11-0153

80. James-Todd TM, Huang T, Seely EW, Saxena AR. The association between phthalates and metabolic syndrome: the National Health and Nutrition Examination Survey 2001-2010. Environ Health. 2016;15:52. doi:10.1186/s12940-016-0136-x

81. Luo K, Zhang R, Aimuzi R, et al. Exposure to organophosphate esters and metabolic syndrome in adults. Environ Int. 2020;143:105941. doi:10.1016/j.envint.2020.105941

82. Lin TW, Chen MK, Lin CC, et al. Association between exposure to perfluoroalkyl substances and metabolic syndrome and related outcomes among older residents living near a Science Park in Taiwan. Int J Hyg Environ Health. 2020;230:113607. doi:10.1016/j.ijheh.2020.113607

83. Zamora AN, Jansen EC, Tamayo-Ortiz M, et al. Exposure to phenols, phthalates, and parabens and development of metabolic syndrome among Mexican Women in midlife. Front Public Health. 2021;9:620769. doi:10.3389/fpubh.2021.620769

84. Nowicki GJ, Ślusarska B, Prystupa A, et al. Assessment of concentrations of heavy metals in postmyocardial infarction patients and patients free from cardiovascular event. Cardiol Res Pract. 2021;2021:9546358. doi:10.1155/2021/9546358

85. Cohen G, Levy I. Chronic exposure to traffic-related air pollution and cancer incidence among 10,000 patients undergoing percutaneous coronary interventions: a historical prospective study. Eur J PrevCardiol. 2018;25(6):659-670. doi:10.1177/ 2047487318760892

86. Eom SY, Choi SH, Ahn SJ, et al. Reference levels of blood mercury and association with metabolic syndrome in Korean adults. Int Arch Occup Environ Health. 2014;87(5):501-513. doi:10.1007/s00420-013-0891-8

87. Xu P, Liu A, Li F, et al. Associations between metabolic syndrome and four heavy metals: a systematic review and meta-analysis. Environ Pollut. 2021;273:116480. doi:10.1016/j. envpol.2021.116480

88. Ma J, Zhou Y, Wang D, et al. Associations between essential metals exposure and metabolic syndrome (MetS): exploring the mediating role of systemic inflammation in a general Chinese population. Environ Int. 2020;140:105802. doi:10.1016/j. envint.2020.105802

89. Zhang JS, Gui ZH, Zou ZY, et al. Long-term exposure to ambient air pollution and metabolic syndrome in children and adolescents: a national cross-sectional study in China. Environ Int. 2021;148:106383. doi:10.1016/j.envint.2021.106383
90. Yang BY, Qian ZM, Li S, et al. Long-term exposure to ambient air pollution (including $\mathrm{PM}_{1}$ ) and metabolic syndrome: the 33 Communities Chinese Health Study (33CCHS). Environ Res. 2018;164:204-211. doi:10.1016/j.envres.2018.02.029

91. Matthiessen C, Lucht S, Hennig F, et al. Long-term exposure to airborne particulate matter and $\mathrm{NO}_{2}$ and prevalent and incident metabolic syndrome - results from the Heinz Nixdorf Recall Study. Environ Int. 2018;116:74-82. doi:10.1016/j. envint.2018.02.035

92. Park SK, Oh CM, Kim MH, et al. Metabolic syndrome, metabolic components, and their relation to the risk of pancreatic cancer. Cancer. 2020;126(9):1979-1986. doi:10.1002/cncr.32737

93. Esposito K, Chiodini P, Colao A, Lenzi A, Giugliano D. Metabolic syndrome and risk of cancer: a systematic review and meta-analysis. Diabetes Care. 2012;35(11):2402-2411. doi: $10.2337 / \mathrm{dc} 12-0336$

94. Venter AC, Roşca E, Daina LG, et al. Phyllodes tumor: diagnostic imaging and histopathology findings. Rom J MorpholEmbryol. 2015;56(4):1397-1402.

95. Jafri H, Alsheikh-Ali AA, Karas RH. Baseline and on-treatment high-density lipoprotein cholesterol and the risk of cancer in randomized controlled trials of lipid-altering therapy. J Am Coll Cardiol. 2010;55(25):2846-2854. doi:10.1016/j.jacc.2009.12.069

96. Stolzenberg-Solomon RZ, Weinstein S, Pollak M, et al. Prediagnostic adiponectin concentrations and pancreatic cancer risk in male smokers. Am J Epidemiol. 2008;168(9):1047-1055. doi:10.1093/aje/kwn221

97. Esposito K, Capuano A, Giugliano D. Metabolic syndrome and cancer: holistic or reductionist? Endocrine. 2014;45(3):362-364. doi:10.1007/s12020-013-0056-2

98. Wagner KH, Brath H. A global view on the development of non communicable diseases. Prev Med. 2012;54(Suppl):S38-S41. doi:10.1016/j.ypmed.2011.11.012

99. Campbell PT. Obesity: a certain and avoidable cause of cancer. Lancet. 2014;384(9945):727-728. doi:10.1016/S0140-6736(14) 61172-7

100. Calle EE, Rodriguez C, Walker-Thurmond K, Thun MJ. Overweight, obesity, and mortality from cancer in a prospectively studied cohort of U.S. adults. $N$ Engl J Med. 2003;348(17):1625-1638. doi:10.1056/NEJMoa021423

101. Scappaticcio L, Maiorino MI, Bellastella G, Giugliano D, Esposito K. Insights into the relationships between diabetes, prediabetes, and cancer. Endocrine. 2017;56(2):231-239. doi:10.1007/s12020-016-1216-y

102. Harding JL, Sooriyakumaran M, Anstey KJ, et al. Hypertension, antihypertensive treatment and cancer incidence and mortality: a pooled collaborative analysis of 12 Australian and New Zealand cohorts. J Hypertens. 2016;34(1):149-155. doi:10.1097/ НJH.0000000000000770

103. Pantyley V. Health inequalities among rural and urban population of Eastern Poland in the context of sustainable development. Ann Agric Environ Med. 2017;24(3):477-483. doi:10.5604/ 12321966.1233905

104. Shin D, Kongpakpaisarn K, Bohra C. Trends in the prevalence of metabolic syndrome and its components in the United States 2007-2014. Int J Cardiol. 2018;259:216-219. doi:10.1016/j. ijcard.2018.01.139

105. Zatońska K, Basiak-Rasała A, Różańska D, et al. Changes in diabetes prevalence and corresponding risk factors - findings from 3- and 6-year follow-up of PURE Poland cohort study. BMC Public Health. 2020;20(1):843. doi:10.1186/s12889-02008970-5 
106. Cukrzyca 2025. Strategia prewencji i leczenia cukrzycy w Polsce. [Diabetes 2025. Strategy for diabetes prevention and treatment in Poland. Available from: http://ippez.pl/wp-content/uploads/2019/ 03/Cukrzyca-20251.pdf. Accessed December 19, 2020.

107. Czauderna P, Gałązka-Sobotka M, Górksi P, Hryniewiecki T Strategiczne kierunki rozwoju system ochrony zdrowia w Polsce. Wyniki ogólnonarodowej debaty o kierunkach zmian w ochronie zdrowia. Dokument podsumowujący. [Strategic directions of development of the health care system in Poland. Results of the nationwide debate on the directions of changes in healthcare.Summary document]. Available from: http://oipip.elblag.pl/wp-content/ uploads $/ 2019 / 07 / \mathrm{Wsp} \% \mathrm{C} 3 \%$ B3lnie-dla-zdrowia_dokument-podsu mowuj\%C4\%85cy.pdf. Accessed December 19, 2020.
108. Dubas K, Seweryn M, Koperny M. Samorządowe programy zdrowotne - praktyczne aspekty realizacji. [Community health programmes - practical aspects of realization]. Hygeia Public Health. 2014;49(4):747-754.

109. Strauß M, Foshag P, Przybylek B, et al. Occupation and metabolic syndrome: is there correlation? A cross sectional study in different work activity occupations of German firefighters and office workers. Diabetol Metab Syndr. 2016;8(1):57. doi:10.1186/ s13098-016-0174-0

\section{Publish your work in this journal}

Diabetes, Metabolic Syndrome and Obesity: Targets and Therapy is an international, peer-reviewed open-access journal committed to the rapid publication of the latest laboratory and clinical findings in the fields of diabetes, metabolic syndrome and obesity research. Original research, review, case reports, hypothesis formation, expert opinion and commentaries are all considered for publication. The manuscript management system is completely online and includes a very quick and fair peer-review system, which is all easy to use. Visit http://www.dovepress.com/testimonials.php to read real quotes from published authors. 